\title{
GENERIC EXISTENCE OF A SOLUTION FOR A DIFFERENTIAL EQUATION IN A SCALE OF BANACH SPACES
}

\author{
TOMÁS DOMÍNGUEZ BENAVIDES
}

\begin{abstract}
Let $\left\{X_{s}: \alpha \leq s \leq \beta\right\}$ be a scale of Banach spaces, $J$ a real interval, $U$ an open subset of $J \times X_{s}$ for some $s$. In this paper we prove that the existence of solutions for

$$
x^{\prime}=A(t) x+f(t, x), \quad x\left(t_{0}\right)=x_{0},
$$
\end{abstract}

is a generic property, when $A(t)$ is an operator satisfying

$$
|A(t)|_{L\left(X_{s^{\prime}} ; X_{s}\right)} \leq M\left(s^{\prime}-s\right)^{-1} \quad\left(M>0 \text { independent of } s, s^{\prime}, t\right)
$$

in the scale $\left\{X_{s}\right\}$ and $f: J \times U \rightarrow X_{\beta}$ is continuous.

1. Introduction. A property is said to be generic in a Baire space $E$ if it holds in a residual subset of $E$. Let $X$ be an infinite dimensional Banach space, $\mathbf{R}$ the set of real numbers and $V$ an open subset of $\mathbf{R} \times X$. Denote by $C(V ; X)$ the set of all continuous mappings from $V$ into $X$, endowed with the topology of uniform convergence. Lasota and Yorke [7] proved that the existence of solutions for the differential equation

$$
x^{\prime}=f(t, x), \quad x\left(t_{0}\right)=x_{0},
$$

is a generic property in $C(V ; X)$. The generic existence of solutions is also studied in $[12,1,3]$ for ordinary differential equations in Banach spaces, and in $[2,4,10]$ for integral and functional equations.

In this paper we study the corresponding problem for differential equations in a scale of Banach spaces. These equations were introduced by Ovcyannikov [8] who proved the existence of solutions for the linear differential equation

$$
x^{\prime}=A(t) x, \quad x\left(t_{0}\right)=x_{0},
$$

where $A(t)$ is a linear operator satisfying conditon (1) (see $\S 2$ ) in a scale $\left\{X_{s}: \alpha \leq\right.$ $s \leq \beta\}$ of Banach spaces. Treves [11] considered the equation

$$
x^{\prime}=A(t) x+g(t), \quad x\left(t_{0}\right)=x_{0},
$$

where $g: J \rightarrow X_{\beta}$ is a continuous mapping on a real interval $J$, and he applied this equation to solve a Cauchy-Kowaleska problem. Deimling [5, p. 26] proved the existence of solutions for the more general equation

$$
x^{\prime}=A(t) x+f(t, x), \quad x\left(t_{0}\right)=x_{0},
$$

Received by the editors January 19, 1981 and, in revised form, November 2, 1981.

1980 Mathematics Subject Classification. Primary 34G20; Secondary 54C50.

Key words and phrases. Differential equation, scale of Banach spaces, Baire category, residual subset, generic property. 
where $f: J \times D \rightarrow X_{\beta}$ is a bounded, uniformly continuous mapping, $\alpha$-Lipschitz with respect to the second variable, and $D$ denotes a closed ball in $X_{s}$ for some fixed $s$ in $(\alpha, \beta)$. Let $U$ be an open subset of $J \times X_{s}$ and denote by $C\left(U ; X_{\beta}\right)$ the set of all continuous mappings from $U$ in $X_{\beta}$ endowed with the topology of uniform convergence. In this paper (Theorem 8 ) we prove that problem (III) has a unique solution for every function $f$ in a residual subset of the Baire space $C\left(U ; X_{\beta}\right)$. In order to better clarify the significance of this theorem, notice that the subset of $C\left(U ; X_{\beta}\right)$ formed by all locally $\alpha$-Lipschitz mappings is of Baire first category $[\mathbf{7}$, Theorem 2] and that for the easier problem (I) there exists a dense subset $\mathcal{E}$ of $C(V ; X)$ such that (I) has no solution for every $f$ in $\mathcal{E}[9]$.

2. Minimal interval of existence of solution and continuous dependence. In order to prove the generic existence of solutions we need to know some fundamental properties of the solutions of (III). In the following we will assume that $\left\{x_{s}: \alpha \leq\right.$ $s \leq \beta\}$ is a scale of Banach spaces such that $X_{s^{\prime}} \subset X_{s}$ and $|x|_{s} \leq|x|_{s^{\prime}}$ for $s<s^{\prime}$ and $x \in X_{s^{\prime}}$, where $|x|_{s}$ denotes norm in $X_{s}$. For some fixed positive number $a$, we will denote by $J$ the interval $\left(t_{0}-a, t_{0}+a\right), t_{0}$ being an arbitrary real number. If $X$ is a metric space, $x$ is in $X$, and $A$ is a subset of $X$, we denote by $d(x, A)$ the number $\inf \{d(x, y): y \in A\}$ and by $B(x, r)(\bar{B}(x, r))$ the open (closed) ball centered at $x$ with radius $r$. Finally if $X, Y$ are metric spaces and $Y$ is complete, $C(X ; Y)$ will denote the complete metric space of all continuous mappings from $X$ into $Y$ with the metric

$$
d(f, g)=\sup \{\min \{1, d(f(x), g(x))\}: x \in X\} .
$$

Lemma 1 [5, TheOREn 1.2]. Let $\left\{X_{s}: \alpha \leq s \leq \beta\right\}$ be a scale of Banach spaces, $g: J \rightarrow X_{\beta}$ continuous, $x_{0} \in X_{3}, A: J \rightarrow L\left(X_{s^{\prime}} ; X_{s}\right)$ a continuous mapping for every pair $\left(s^{\prime}, s\right), \alpha \leq s<s^{\prime} \leq 3$. satisfying

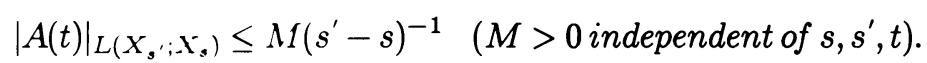

Then for every $s \in[\alpha, \beta)$ problem $(\Pi)$ has a unique solution $\phi:\left(t_{0}-\delta, t_{0}+\delta\right) \rightarrow X_{s}$ where $\delta=\min \{a,(\beta-s) / M e\}$ and

$$
\begin{aligned}
\left|\phi(t)-x_{0}\right|_{s} \leq & \left(\left|x_{0}\right|_{\beta}+(\beta-s) M^{-1} \max _{\left[t_{0}, t\right] \text { or }\left[t, t_{0}\right]}|g(\tau)|_{\beta}\right) \\
& \times M e\left|t-t_{0}\right|\left(\beta-s-M e\left|t-t_{0}\right|\right)^{-1} .
\end{aligned}
$$

Let $A(t)$ be as in Lemma $1, \Omega$ an open subset of $X_{s}, x_{0}$ a point in $\Omega \cap X_{\beta}$, $f: J \times \Omega \rightarrow X_{\beta}$ continuous and $\phi$ a solution of (III) defined on an interval $\left(\tau_{1}, \tau_{2}\right)$ contained in $J$. We recall [7] that $\phi$ is said to be unlimited if there does not exist $\lim (t, \phi(t))$ as $t \rightarrow \tau_{i}(i=1,2)$ in $J \times \Omega$.

THEOREM 2. Let $A(t), x_{0}$ be as in Lemma $1, \Omega$ an open subset of $X_{s}$ which contains $x_{0}, f: J \times \Omega \rightarrow X_{\beta}$ continuous and bounded, and $c$ a bound for $f$.

(a) If $\phi$ is a solution of (III) defined on $l=\left[t_{0}-b, t_{0}+b\right]$ for some $b<$ $\min \{a,(\beta-s) / M e\}$, then $\phi(t)$ is in $X_{s^{\prime}}$ for every $t$ in $l$ and $s^{\prime} \in[s, \beta-M e b)$. Furthermore one has

$$
\left|\phi(t)-x_{0}\right|_{s^{\prime}} \leq N\left(s^{\prime}, b, x_{0}\right)=\left(\left|x_{0}\right|_{\beta}+c(\beta-s) M^{-1}\right) M e b\left(\beta-s^{\prime}-M e b\right)^{-1},
$$

and for every $\epsilon<\beta-s^{\prime}-$ Meb one has

$$
|\phi(t)-\phi(\bar{t})|_{s^{\prime}} \leq P\left(s^{\prime}, \epsilon, b, x_{0}\right)|t-\bar{t}| \quad(t, \bar{t} \in l),
$$


where $P\left(s^{\prime}, \epsilon, b, x_{0}\right)=\left(\left|x_{0}\right|_{\beta}+N\left(s^{\prime}+\epsilon, b, x_{0}\right)\right)\left(M \epsilon^{-1}+c\right)$.

(b) If $\psi$ is an unlimited solution of (III), then $\psi$ is defined at least on the interval $\left(t_{0}-\delta, t_{0}+\delta\right), \delta=\min \{a,(\beta-s) /$ Mhe $\}$ where

$$
h=\left\{\begin{array}{l}
1 \text { if } d=d\left(x_{0}, \Omega^{c}\right)=+\infty, \\
\left(\left|x_{0}\right|_{\beta}+c M^{-1}(\beta-s)+d\right) d^{-1} \text { otherwise. }
\end{array}\right.
$$

Proof. (a) Define $g(t)=f(t, \phi(t)), t \in l$. From Lemma 1 we know that for every $s^{\prime} \in[s, \beta-M e b)$ problem (II) has a unique solution $\bar{\phi}:\left(t_{0}-\delta^{\prime}, t_{0}+\delta^{\prime}\right) \rightarrow X_{s^{\prime}}$ where $\delta^{\prime}=\min \left\{a,\left(\beta-s^{\prime}\right) / M e\right\}>b$. Since $\phi$ and $\bar{\phi}$ are solutions of (II) in $X_{s}$ we derive from Lemma 1 that $\phi(t)=\bar{\phi}(t)$ for every $t$ in $\left(t_{0}-\delta^{\prime}, t_{0}+\delta^{\prime}\right)$. Thus $\phi(t)$ belongs to $X_{s^{\prime}}$ for every $s^{\prime} \in[s, \beta-M e b)$. Inequality (3) follows quite immediately from (2). Condition (1) for $\left(s^{\prime}, s^{\prime}+\epsilon\right)$, applied to the integral equation for (III), implies (4).

(b) Assume $\psi$ is defined on $\left(\tau_{1}, \tau_{2}\right)$ where $\tau=\min \left\{\left|t_{0}-\tau_{1}\right|,\left|t_{0}-\tau_{2}\right|\right\}<\delta$. Since $\psi$ is defined on $\left(t_{0}-\tau, t_{0}+\tau\right)$ we obtain from (4)

$$
|\psi(t)-\psi(\bar{t})|_{s} \leq P\left(s+\epsilon, \epsilon, \tau, x_{0}\right)|t-\bar{t}| \quad\left(t, \bar{t} \in\left(t_{0}-\tau, t_{0}+\tau\right)\right)
$$

for some $\epsilon<\beta-s-M e \tau$, i.e. $\psi$ satisfies a Cauchy condition. Hence there exists $\lim \psi\left(t_{0}+t\right)=\rho$ as $t \rightarrow \tau$ or $t \rightarrow-\tau$, and we claim that $\rho$ is in $\Omega$. Indeed, from (3) we derive $\left|\rho-x_{0}\right|_{s} \leq N\left(s, \tau, x_{0}\right)$. Using $\beta-s \geq h \delta M e>h \tau M e$ and the definition of $h$ for $d<+\infty$ we have

$$
\left|\rho-x_{0}\right|_{s}<\left(\left|x_{0}\right|_{\beta}+c M^{-1}(\beta-s)\right)(h-1)^{-1}=d .
$$

LEMMA 3. Let $A(t), x_{0}$ be as in Lemma 1 and assume the following hold:

(i) $D=\bar{B}\left(x_{0}, R\right)$ is a closed ball in $X_{s}$ for some fixed $s \in(\alpha, \beta)$.

(ii) $f_{0}: J \times D \rightarrow X_{\beta}$ is continuous, bounded and Lipschitz-continuous with respect to the second variable with modulus $L$.

(iii) $\left\{\left(t_{n}, x_{n}\right)\right\}$ is a sequence which converges to $\left(t_{0}, x_{0}\right)$ in $J \times X_{\beta}$.

(iv) $\left\{f_{n}\right\}$ is a sequence which converges to $f_{0}$ in $C\left(J \times D ; X_{\beta}\right)$.

(v) For every nonnegative integer $n, \phi_{n}$ is an unlimited solution of

$$
x^{\prime}=A(t) x+f_{n}(t, x), \quad x\left(t_{n}\right)=x_{n} .
$$

Let $c$ be any real number greater than $\sup \left\{|f(t, x)|_{\beta}:(t, x) \in J \times D\right\}$ and set $h=$ $2\left(\left|x_{0}\right|_{\beta}+R+c(\beta-s) M^{-1}\right) R^{-1}$. Then there exists a positive integer $n_{0}$ such that for every $\gamma, 0<\gamma \leq \min \left\{a / 4,(\beta-s) / 4 h M e,(4 L e)^{-1}\right\}$ and for every $n \geq n_{0}, \phi_{n}$ is defined from $l=\left[t_{0}-\gamma, t_{0}+\gamma\right]$ into $X_{s^{\prime}}$ and $\left\{\phi_{n}\right\} \rightarrow \phi_{0}$ in $c\left(l ; X_{s^{\prime}}\right)$ for every $s^{\prime}, s \leq s^{\prime}<\beta-3 M e \gamma$.

Proof. Assume $n$ large enough so that $\left|f_{n}(t, x)\right|_{\beta} \leq c$ and $\left|x_{n}-x_{0}\right|_{\beta}<R / 2$. Since $\left|x_{n}\right|_{\beta}<\left|x_{0}\right|_{\beta}+2^{-1} R$ and $d\left(x_{n}, D^{c}\right)>2^{-1} R$ we obtain from Theorem 2(b) that $\phi_{n}$ is defined on $J \cap\left(t_{n}-4 \gamma, t_{n}+4 \gamma\right)$. Hence for $n$ large enough, $\phi_{n}$ is defined on $l$. Set $\gamma^{\prime}=\gamma+\left|t_{n}-t_{0}\right|$. Since $l$ is contained in $\left[t_{n}-\gamma^{\prime}, t_{n}+\gamma^{\prime}\right]$ we deduce from Theorem 2(a) that $\phi_{n}(t)$ belongs to $X_{s^{\prime}}(t \in l)$ for every $s^{\prime}<\beta-M e \gamma^{\prime}$. Thus for $n$ large enough so that $\left|t_{n}-t_{0}\right|<\gamma$, say $n \geq n_{0}$, we have that $\phi_{n}(t)$ belongs to $X_{s^{\prime}}$ $\left(t \in l, n \geq n_{0}\right)$ for every $s^{\prime}<\beta-2 M e \gamma$. Denote by $D_{s^{\prime}}$ the closed set $D \cap X_{s^{\prime}}$ in $X_{s^{\prime}}$ and define for every $u$ in $C\left(l ; D_{s^{\prime}}\right)$ a mapping $T_{n} u\left(n \geq n_{0}\right)$ by: $T_{n} u$ is the unique solution on $l$ of

$$
x^{\prime}=A(t) x+f_{n}(t, u(t)), \quad x\left(t_{n}\right)=x_{n} .
$$


Notice that Lemma 1 assures the existence and uniqueness of $T_{n} u$ : Furthermore by Theorem 2(a), $T_{n} u(t)$ belongs to $X_{s^{\prime}}$ for every $t$ in $l$ and $s^{\prime}<\beta-2 M e \gamma<\beta-M e \gamma^{\prime}$.

ClaIM 1. $T_{n} u$ belongs to $C\left(l ; D_{s^{\prime}}\right)$ for every $u$ in $C\left(l ; D_{s^{\prime}}\right), s \leq s^{\prime}<\beta-2 M e \gamma$, $n \geq n_{0}$.

Choose $\epsilon<\beta-s^{\prime}-2 M e \gamma<\beta-s^{\prime}-M e \gamma^{\prime}$. From (4) we have

$$
\left|T_{n} u(t)-T_{n} u(t)\right|_{s^{\prime}} \leq P\left(s^{\prime}, \epsilon, \gamma^{\prime}, x_{n}\right)|t-\bar{t}| .
$$

Thus $T_{n} u$ is a continuous mapping. Hence it suffices to prove that $T_{n} u(t)$ is in $D$ for every $t \in l$. From (3) we have

$$
\left|T_{n} u(t)-x_{n}\right|_{s} \leq N\left(s, \gamma^{\prime}, x_{n}\right)<N\left(s, 2 \gamma, x_{n}\right) .
$$

Using $\left|x_{n}\right|_{\beta}<\left|x_{0}\right|_{\beta}+R / 2, \beta-s \geq 2 h M e \gamma$, the definition of $h$, and following an argument as in the final part of Theorem 2 we derive $N\left(s, 2 \gamma, x_{n}\right)<R / 2$. Hence $\left|T_{n} u(t)-x_{0}\right|_{\beta}<R$.

ClaIM 2. For $u$ in $C(l ; D)$ denote $\|u\|=\sup \left\{|u(t)|_{s}: t \in l\right\}$. Then for every $s^{\prime}$, $s \leq s^{\prime}<\beta-2 M e \gamma, u, v$ in $C\left(l ; D_{s^{\prime}}\right)$ and $t \in l$ we have $\left|T_{0} u(t)-T_{0} v(t)\right|_{s^{\prime}} \leq 2^{-1}\|u-v\|$.

Since $T_{0} u-T_{0} v$ is a solution of the equation

$$
x^{\prime}=A(t) x+f_{0}(t, u(t))-f_{0}(t, v(t)), \quad x\left(t_{0}\right)=x_{0},
$$

we obtain from (2)

$$
\left|T_{0} u(t)-T_{0} v(t)\right|_{s^{\prime}} \leq\left(\beta-s^{\prime}\right) M^{-1} L \max _{\tau \in l}|u(\tau)-v(\tau)|_{s} M e \gamma\left(\beta-s^{\prime}-M e \gamma\right)^{-1}
$$

that is less than $2^{-1}\|u-v\|$. Here we use $-M e \gamma \geq-\left(\beta-s^{\prime}\right) / 2$ and the definition of $\gamma$.

ClaIM 3. For every $s, s \leq s^{\prime}<\beta-3 \mathrm{Me \gamma}$ the sequence $\left\{T_{n}\right\}$ converges to $T_{0}$ uniformly on $C\left(l ; D_{s^{\prime}}\right)$.

Let $s^{\prime}$ satisfy $s \leq s^{\prime}<\beta-3 M e \gamma$ and choose $s^{\prime \prime}<\beta-2 M e \gamma$ such that $s^{\prime \prime}-s^{\prime}>$

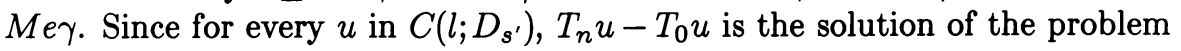

$$
\begin{aligned}
x^{\prime} & =A(t) x+f_{n}(t, u(t))-f_{0}(t, u(t)) ; \\
x\left(t_{0}\right) & =y_{n}=x_{n}-x_{0}+\int_{t_{n}}^{t_{0}}\left[A(\tau) T_{n} u(\tau)+f_{n}(\tau, u(\tau))\right] d \tau
\end{aligned}
$$

in the scale $\left\{X_{r}: \alpha \leq r \leq s^{\prime \prime}\right\}$ (notice that $y_{n}$ belongs to $X_{s^{\prime \prime}}$ ) we obtain from (2),

$$
\begin{aligned}
\mid T_{n} u(t)- & \left.T_{0} u(t)\right|_{s^{\prime}} \\
\leq & \left|y_{n}\right|_{s^{\prime}}+\left(\left|y_{n}\right|_{s^{\prime \prime}}+M^{-1}\left(s^{\prime \prime}-s^{\prime}\right) \max _{t \in l}\left|f_{0}(t, u(t))-f_{n}(t, u(t))\right|_{s^{\prime \prime}}\right) \\
& \times \operatorname{Me\gamma }\left(s^{\prime \prime}-s^{\prime}-M e \gamma\right)^{-1} .
\end{aligned}
$$

Since $\left\{f_{n}\right\} \rightarrow f_{0}$ uniformly, it suffices to prove that $\left|y_{n}\right|_{s^{\prime \prime}} \rightarrow 0$ uniformly with respect to $u$ in $C\left(l ; D_{s^{\prime}}\right)$. From (3) there exists a constant $N$ such that $\left|T_{n} u(t)\right|_{s^{\prime \prime}+\epsilon}<$ $N$ for some small $\epsilon>0$ and for every $u$ in $C\left(l ; D_{s^{\prime}}\right), t \in l$. Hence condition (1) applied to $\left(s^{\prime \prime}, s^{\prime \prime}+\epsilon\right)$ implies

$$
\left|y_{n}\right|_{s^{\prime \prime}} \leq\left|x_{n}-x_{0}\right|_{s^{\prime \prime}}+\left|t_{n}-t_{0}\right|\left(M N \epsilon^{-1}+c\right)
$$

and the right-hand side of this inequality converges to 0 and is independent of $u$.

To complete the proof of the lemma, fix $s^{\prime}, s \leq s^{\prime}<\beta-3 \mathrm{Me \gamma}$. Let $\eta$ be an arbitrary positive number and $n_{0}$ large enough so that $\left|T_{n} u(t)-T_{0} u(t)\right|_{s^{\prime}}<\eta / 2$ for 
every $n \geq n_{0}, t \in l$ and $u \in C\left(l ; D_{s^{\prime}}\right)$. Since $\phi_{n}$ is a fixed point of $T_{n}$ we have

$$
\begin{aligned}
\sup _{t \in l}\left|\phi_{n}(t)-\phi_{0}(t)\right|_{s^{\prime}} & =\sup _{t \in l}\left|T_{n} \phi_{n}(t)-T_{0} \phi_{0}(t)\right|_{s^{\prime}} \leq 2^{-1}\left\|\phi_{n}-\phi_{0}\right\|+\eta / 2 \\
& \leq 2^{-1} \sup _{t \in l}\left|\phi_{n}(t)-\phi_{0}(t)\right|_{s^{\prime}}+\eta / 2 .
\end{aligned}
$$

Therefore $\left\{\phi_{n}\right\} \rightarrow \phi_{0}$ in $C\left(l ; D_{s^{\prime}}\right)$.

THEOREM 4. Let $A(t)$ be as in Lemma 1, and assume the following hold:

(i) $\Omega$ is an open subset of $X_{s}$ for some fixed $s \in(\alpha, \beta)$.

(ii) $f_{0}: J \times \Omega \rightarrow X_{\beta}$ is locally Lipschitz-continuous and bounded.

(iii) $\left\{f_{n}\right\}$ is a sequence which converges to $f_{0}$ in $C\left(J \times \Omega, X_{\beta}\right)$.

(iv) $\left\{\left(t_{n}, x_{n}\right)\right\}$ is a sequence which converges to $\left(t_{0}, x_{0}\right)$ in $J \times X_{\beta}$.

(v) For any nonnegative integer $n, \phi_{n}$ is an unlimited solution of (IV).

Then, for every real number $b<\delta$ where $\delta=\min \{a,(\beta-s) / M h e\}, h$ defined as in Theorem 2, one has: There exists a positive integer $n_{0}$ such that for every $n \geq n_{0}$, $\phi_{n}$ is defined on $l=\left[t_{0}-b, t_{0}+b\right]$ and $\left\{\phi_{n}\right\}$ converges to $\phi_{0}$ in $C\left(l ; X_{s^{\prime}}\right)$ for every $s^{\prime}$, $s \leq s^{\prime}<\beta-M e b$.

PROOF. From Theorem 2(b), $\phi_{0}$ and $\phi_{n}$ are defined on $l$ for $n$ large enough. Using Theorem 2(a) it is easy to prove that $\phi_{0}(t)$ and $\phi_{n}(t)$ belong to $X_{s^{\prime}}$ for every $t \in l, s^{\prime} \in[s, \beta-M e b)$ and $n$ large enough, say $n \geq n_{0}$. Let $s_{1}, s_{2}$ be real numbers, $s \leq s_{1}<s_{2}<\beta-M e b$. It is easy to prove from (4) that there exist a constant $P$, independent of $n$, such that

$$
\left|\phi_{n}(t)-\phi_{n}(\bar{t})\right|_{s_{2}} \leq P|t-\bar{t}|, \quad t, \bar{t} \in l, \quad n \geq n_{0} .
$$

Define the set $S=\left\{t \leq b:\left\{\phi_{n}\right\} \rightarrow \phi_{0}\right.$ in $C\left(\left[t_{0}-t, t_{0}+t\right] ; X_{s^{\prime}}\right)$ for some $s^{\prime}, s_{1}<$ $\left.s^{\prime} \leq s_{2}\right\}$. Since zero is in $S$ we have $S \neq \varnothing$. Furthermore, the equicontinuity condition (5) implies that $S$ is a closed set. Set $\sigma=\sup S$. Then $\left\{\phi_{n}\right\} \rightarrow \phi_{0}$ in $C\left(\left[t_{0}-\sigma, t_{0}+\sigma\right] ; X_{s^{\prime}}\right)$ for some $s^{\prime}, s_{1}<s^{\prime} \leq s_{2}$. We claim that $\sigma=b$. Otherwise set $\Omega_{1}=\Omega \cap X_{s_{1}}$ and notice that $f_{0}: J \times \Omega_{1} \rightarrow X_{\beta}$ satisfied the hypotheses of Lemma 3 in a neighborhood of $\left(t_{0}+\sigma, \phi_{0}\left(t_{0}+\sigma\right)\right)$ for the sequences $\left\{f_{n}\right\} \rightarrow f_{0}$ in $C\left(J \times \Omega_{1} ; X_{\beta}\right)$ and $\left\{\left(t_{0}+\sigma, \phi_{n}\left(t_{0}+\sigma\right)\right)\right\}$ converging to $\left(t_{0}+\sigma, \phi_{0}\left(t_{0}+\sigma\right)\right)$ in $X_{s^{\prime}}$. Therefore we can apply Lemma 3 (where $s$ is replaced by $s_{1}$, and $\beta$ by $s^{\prime}$ ) to prove that $\left\{\phi_{n}\right\} \rightarrow \phi_{0}$ in $C\left(\left[t_{0}+\sigma-\gamma, t_{0}+\sigma+\gamma\right] ; X_{s^{\prime \prime}}\right)$ for some $\gamma>0$ and $s^{\prime \prime}, s_{1}<s^{\prime \prime}<s^{\prime}$. Since the same argument holds for the point $\left(t_{0}-\sigma, \phi_{0}\left(t_{0}-\sigma\right)\right)$ we get a contradiction. Thus $\left\{\phi_{n}\right\} \rightarrow \phi_{0}$ in $C\left(l ; X_{s^{\prime}}\right)$ for some $s^{\prime}>s_{1}$ and "a fortiori" in $C\left(l ; X_{s_{1}}\right)$.

3. Generic existence of solutions. To prove the generic existence of solutions of (3) we follow the pattern developed in [7] for ordinary differential equations. In the following $U$ will denote a subset of $\mathbf{R} \times X_{s}$ for some fixed $s \in(\alpha, \beta), u_{0}=$ $\left(t_{0}, x_{0}\right)$ a point in $U\left(x_{0} \in X_{\beta}\right)$ and $X$ the Baire space $C\left(U ; X_{\beta}\right)$. If $u=(t, x)$ and $\bar{u}=(\bar{t}, \bar{x})$ are in $U d(u, \bar{u})$ will mean $\max \left\{|x-\bar{x}|_{s},|t-\bar{t}|\right\}$. For some number $k$, $0<k<1$, choose a number $\bar{s}$ in $(s, k s+(1-k) \beta)$. Let $f$ be a mapping in $X$, $c_{f}^{\prime}=\left|f\left(u_{0}\right)\right|_{\beta}+1, U_{f}=\operatorname{int}\left\{(t, x) \in U:|f(t, x)|_{\beta} \leq c_{f}^{\prime}\right\}, c_{f}=c_{f}^{\prime}+1, D_{f}=d\left(u_{0}, U_{f}^{c}\right)$ and $\delta_{f}=\min \left\{D_{f},(\beta-s) / M e h_{f}\right\}$ where

$$
h_{f}=\left\{\begin{array}{l}
1 \text { if } D_{f}=+\infty, \\
\left(\left|x_{0}\right|_{\beta}+c_{f} M^{-1}(\beta-s)+D_{f}\right) D_{f}^{-1} \text { if } D_{f}<+\infty .
\end{array}\right.
$$

Denote by $K_{f}$ the interval $\left(t_{0}-\delta_{f}, t_{0}+\delta_{f}\right)$ and by $J_{f}$ the interval $\left[t_{0}-k \delta_{f}, t_{0}+k \delta_{f}\right]$. Notice that $\bar{s}$ is in $\left(s, \beta-M e k \delta_{f}\right)$ for every $f$ in $X$. 
Let $f_{1}, f_{2}$ be mappings in $X ; u_{i}=\left(t_{i}, x_{i}\right)(1=1,2)$ points in $J \times X_{\beta}$. If $\phi_{i}$ $(i=1,2)$ are solutions in $X_{\bar{s}}$ of

$$
x^{\prime}=A(t) x+f_{i}(t, x), \quad x\left(t_{i}\right)=x_{i},
$$

defined on $J_{f}$ we denote

$$
\begin{gathered}
d_{f}\left(\phi_{1}, \phi_{2}\right)=\sup \left\{\left|\phi_{1}(t)-\phi_{2}(t)\right|_{\bar{s}}: t \in J_{f}\right\}, \\
\mu(f, \delta)=\sup \left\{d_{f}\left(\phi_{1}, \phi_{2}\right): f_{1} \in B(f, \delta) ; u_{i} \in B\left(u_{0}, \delta\right),\right. \\
\left.\phi_{i} \text { unlimited solutions of (VI) defined on } J_{f}\right\}, \\
V(f)=\lim \sup \mu(f, \delta) \text { as } \delta \rightarrow 0 .
\end{gathered}
$$

LEMMA 5. Let $f$ be a mapping in $X$ such that $V(f)=0$. Then

(i) there exists a solution $\phi: J_{f} \rightarrow X_{s}$ of (III);

(ii) if $\bar{\phi}$ is another solution of (III) defined on $J_{f}$, then $\phi(t)=\bar{\phi}(t)$ for every $t$ in $J_{f}$;

(iii) the solution $\phi$ depends continuously on $f$ and $u$; i.e. if $\left\{f_{n}\right\} \rightarrow f$ in $X,\left\{u_{n}\right\} \rightarrow$ $u_{0}$ in $J \times X_{\beta}$ and $\phi_{n}$ is an unlimited solution of (IV), then there exist a positive integer $n_{0}$, such that $\phi_{n}$ is defined on $J_{f}$ if $n \geq n_{0}$ and $\left\{\phi_{n}\right\} \rightarrow \phi$ in $C\left(J_{f} ; X_{s}\right)$.

PROOF. From [7, Lemma 1] we can choose a sequence of locally Liptschitz mappings $\left\{f_{n}\right\}$ which converges to $f$ in $X$ assuming without loss of generality $\left|f_{n}(t, x)\right|_{\beta}<c_{f}$ on $U_{f}$. From Theorem 2 the unique unlimited solution $\psi_{n}$ of (IV) (whose existence is given by [5, Theorem 2.4] is defined from $J_{f}$ into $X_{s^{\prime}}, s^{\prime} \in$ $\left[s, \beta-M e k \delta_{f}\right)$ for $n$ large enough. Since $V(f)=0$ we derive that $\left\{\psi_{n}\right\}$ is a Cauchy sequence in $C\left(J_{f} ; X_{\bar{s}}\right)$ and thus $\left\{\psi_{n}\right\}$ converges in $C\left(J_{f} ; X_{\bar{s}}\right)$ to a mapping $\phi$. Using the integral equation for (IV) we obtain that $\phi$ is a solution of (III) in $X_{s}$. (Recall that $A(t)$ is a continuous operator from $X_{\bar{s}}$ into $X_{s}$.) Since $V(f)=0$ it is easy to prove (ii). In order to prove (iii) notice that $\phi_{n}$ is defined on $J_{f}$ for large $n$. Then $V(f)=0$ implies that $\left\{\phi_{n}\right\} \rightarrow \phi$ in $C\left(J_{f} ; X_{\bar{s}}\right)$ and "a fortiori" in $C\left(J_{f} ; X_{s}\right)$.

The next lemma follows immediately from Theorem 4.

LEMMA 6. Let $f$ be a locally Lipschitz mapping in $X$. Then $V(f)=0$.

LEMMA 7. $V: X \rightarrow \mathbf{R}^{+}$is continuous on the set $V^{-1}(\{0\})$.

Proof. Assume $V(f)=0$. If $V$ is not continuous at $f$, there exists a positive number $\eta$ and a sequence $\left\{f_{n}\right\} \rightarrow f$ such that $V\left(f_{n}\right)>\eta$. For each $n$ there exists a sequence of positive numbers $\delta_{m n} \rightarrow 0, \delta_{m n}<1 / m$, such that $\mu\left(f_{n}, \delta_{m n}\right)>\eta / 2$. Hence there are sequences $\left\{\phi_{i m n}\right\},\left\{f_{i m n}\right\},\left\{u_{i m n}\right\}(i=1,2)$ such that $\phi_{i m n}$ is an unlimited solution of $x^{\prime}=A(t) x+f_{i m n}(t, x), x\left(t_{i m n}\right)=x_{i m n}(i=1,2)$ defined on $J_{f_{n}}, f_{i m n}$ is in $B\left(f_{n}, \delta_{m n}\right), u_{i m n}$ is in $B\left(u_{n}, \delta_{m n}\right)$ and $d_{f_{n}}\left(\phi_{1 m n}, \phi_{2 m n}\right)>\eta / 2$. Since $\delta_{m n}<1 / m$ the diagonal sequences $\left\{f_{i n n}\right\},\left\{u_{i n n}\right\}$ converge to $f$ and $u_{0}$ respectively, and $d_{f_{n}}\left(\phi_{1 n n}, \phi_{2 n n}\right)>\eta / 2$. We will now prove that $d_{f}\left(\phi_{1 n n}, \phi_{2 n n}\right)>\eta / 4$, which is a contradiction because $V(f)=0$. Since the unlimited solutions $\phi_{i n n}$ are defined on $J_{f}$ for $n$ large enough, it suffices to prove

$$
\begin{aligned}
& \sup \left\{\left|\phi_{1 n n}(t)-\phi_{2 n n}(t)\right|_{\bar{s}}: t \in J_{f_{n}}-J_{f}\right\} \\
& \quad \leq \sup \left\{\left|\phi_{1 n n}(t)-\phi_{2 n n}(t)\right|_{\bar{s}}: t \in J_{f}\right\}+\eta / 4 .
\end{aligned}
$$

To prove (6) we let $\theta$ be any positive number. If $D_{f}=d\left(u_{0}, U^{c}\right)$ it is clear that $D_{f_{n}} \leq D_{f}$ for every $n$ and therefore $D_{f_{n}}<D_{f}+\theta$. If $D_{f}<d\left(u_{0}, U^{c}\right)$ there exists a 
point $u_{1}$ in $U$ such that $d\left(u_{0}, u_{1}\right)<D_{f}+\theta / 2$ and $u_{1}$ is in $U_{f}^{c}$. Hence there exists $\bar{u}$ in the ball $B\left(u_{1}, \theta / 2\right)$ such that $|f(\bar{u})|_{\beta}>\left|f\left(u_{0}\right)\right|_{\beta}+1$. Since $\left\{f_{n}\right\} \rightarrow f$ we can find $n_{0}$ large enough so that $\left|f_{n}(\bar{u})\right|_{\beta}>\left|f_{n}\left(u_{0}\right)\right|_{\beta}+1$ for $n \geq n_{0}$, which implies $D_{f_{n}} \leq d\left(u_{0}, \bar{u}\right)<D_{f}+\theta$. Therefore for every $\epsilon>0$ we have $\delta_{f_{n}}<\delta_{f}+\epsilon$ for $n$ large enough. From (4) it is easy to prove that there exists a constant $P$ independent of $n$, such that

$$
\left|\phi_{i n n}(t)-\phi_{i n n}(\bar{t})\right|_{\bar{s}} \leq P|t-\bar{t}| \quad\left(t, \bar{t} \in J_{f_{n}}\right) .
$$

Assume $n$ is large enough so that $\delta_{f_{n}}-\delta_{f}<\eta(8 P)^{-1}$. Since for every $\bar{t}$ in $J_{f_{n}}-J_{f}$ there exists $t \in J_{f}$ such that $|\bar{t}-t|<\eta(8 P)^{-1}$, it is easy to prove (6) from (7).

THEOREM 8. Let $A$ be the subset of $X$ formed by all mappings which satisfy

(A) problem (3) has a unique solution $\phi$ that is defined at least on $K_{f}$;

(B) if $\left\{f_{n}\right\} \rightarrow f$ in $X,\left\{u_{n}\right\} \rightarrow u_{0}$ in $J \times X_{\beta}$ and (4) has an unlimited solution $\phi_{n}$ then for every compact set $K \subset K_{f}$ there exists a positive integer $n_{0}$ such that $\phi_{n}$ is defined on $K$ for $n \geq n_{0}$ and $\left\{\phi_{n}\right\} \rightarrow \phi$ in $C\left(K ; X_{s}\right)$.

Then $A$ is a residual subset of $X$.

Proof. Let $n$ be any positive integer. From Lemma 7 we see that $V^{-1}([0,1 / n])$ contains an open set $G_{n}$ which contains $V^{-1}(\{0\})$. Then the set $A_{k}=\bigcap_{n=1}^{\infty} G_{n}$ (recall that $V$ depends on a constant $k, 0<k<1$ ) is a dense $G_{\delta}$ in $X$, because locally Lipschitz mappings are dense in $X$ [7, Lemma 2]. Furthermore, if $f$ is in $A_{k}$ we have $V(f)=0$. Consequently, by Lemma $5 f$ satisfies (A) and (B) when $K_{f}$ is replaced by $J_{f}$.

Consider a sequence $\left\{k_{n}\right\} \rightarrow 1$. Then $A=\bigcap_{n=1}^{\infty} A_{k_{n}}$ is also a dense $G_{\delta}$ and every $f$ in $A$ satisfies (A) and (B) on $K_{f}$.

Generic existence also can be studied in the set $U \times X$. In this case we define for every pair $(u, f) \in U \times X$ the function $W: U \times X \rightarrow \mathbf{R}$ by $W(u, f)=V(f)$ for the initial point $u$. The arguments in Lemmas 5-7 apply equally well and we can state

THEOREM 9. Let $B$ be the subset of $U \times X$ formed by all pairs $(u, f)$ which satisfy (A) and (B) for the mapping $f$ and the initial condition $u$. Then $B$ is a residual subset of $U \times X$.

When $U$ is separable we can apply a result of Kuratowski-Ulam [6] and state

THEOREM 10. There exists a residual subset $A$ of $X$ such that for every $f$ in $A$ there exists a residual subset $U^{\prime}$ of $U$ such that (A) and (B) are satisfied for the mapping $f$ and every initial condition $u$ in $U^{\prime}$.

\section{REFERENCES}

1. F. S. De Blasi and J. Myjak, Generic properties of differential equations in a Banach space, Bull. Acad. Polon. Sci. Sér. Sci. Math. 26 (1978), 395-400.

2. _. Some generic properties of functional differential equations in a Banach space, J. Math. Anal. Appl. 67 (1978), 437-451.

3. __ Orlicz type category results for differential equations in Banach spaces, Comment. Math. Prace Mat. (to appear).

4. F. S. De Blasi, M. Kwapisz and J. Myjak, Generic properties of functional equations, Nonlinear Anal. 2 (1977), 239-249. 
5. K. Deimling, Ordinary differential equations in Banach spaces, Lecture Notes in Math., vol. 596, Springer-Verlag, Berlin and New York, 1977.

6. K. Kuratowski and M. Ulam, Quelques propriétés topologiques du produit combinatoire, Fund. Math. 19 (1932), 248-251.

7. A. Lasota and J. A. Yorke, The generic property of existence of solutions of differential equations in Banach spaces, J. Differential Equations 13 (1973), 1-12.

8. L. V. Ovcyannikov, Singular operators in Banach scales, Soviet Math. Dokl. 6 (1965), 10251028.

9. G. Pianigiani, A density result of differential equations in Banach spaces, Bull. Acad. Polon. Sci. Ser. Sci. Math. 26 (1978), 791-793.

10. S. Szufla, The generic property of existence of solutions of integral equations in Banach spaces, Math. Nachr. 93 (1979), 305-312.

11. F. Treves, Basic linear partial differential equation, Academic Press, New York, 1975.

12. G. Vidossich, Existence, uniqueness and approximation of fixed points as a generic property, Bol. Soc. Brasil. Mat. 5 (1974), 17-29.

Facultad de Matemáticas, Universidad de Sevilla, Sevilla, Spain 